\title{
TESTE DE BENDER - SISTEMA DE PONTUAÇÃO GRADUAL: SCOPING REVIEW
}

Fernanda Otoni

Universidade São Francisco, Campinas - SP

Adriana Satico Ferraz

Universidade São Francisco, Campinas - SP

Fabian J. M. Rueda

Universidade São Francisco, Campinas - SP
Recebido em: 26/03/2020

$1^{\text {a }}$ revisão em: 28/06/2021

Aceito em: 08/08/2021

\section{RESUMO}

O teste de Bender avalia a maturidade perceptomotora de crianças a partir da cópia de desenhos. Atualmente no Brasil, o único sistema favorável para correção deste teste é o Sistema de Pontuação Gradual/B-SPG, que considera os erros a partir das distorções da forma. Devido à sua contribuição para a compreensão das dificuldades nas habilidades perceptomotoras apresentadas pela criança e para a elaboração/acompanhamento de intervenções, a presente revisão (scoping review) apresenta um panorama acerca do B-SPG (2005-2020). Foram objeto de análise quantitativa e qualitativa 35 artigos empíricos recuperados do Portal de Periódicos CAPES, Redalyc e Google Scholar. Os resultados indicaram que o B-SPG possui um acúmulo satisfatório de propriedades psicométricas; a sua aplicação em contexto de pesquisa amplia as noções sobre o funcionamento da maturidade perceptomora de crianças brasileiras e que este sistema de correção do Bender também corrobora a prática de psicólogas atuantes nos campos da Psicologia e da Educação.

Palavras-chave: maturidade perceptomotora; avaliação psicoeducacional infantil; prática clínica; desenvolvimento cognitivo; intervenção. 


\section{BENDER TEST - GRADUAL SCORING SYSTEM: SCOPING REVIEW}

\section{ABSTRACT}

Bender test assesses the perceptomotor maturity of children from drawing reproduction. Currently, in Brazil, the only favorable system for correcting this test is the Gradual Scoring System/B-SPG, which considers errors distortions in drawing reproduction. Due to contribution to the comprehension of the difficulties in the perceptomotor skills presented by the child and the elaboration/follow-up of interventions, this scoping review gives an overview of the B-SPG (2005-2020). It was the object of quantitative and qualitative analysis 35 articles empirical recovered from the CAPES Journal Portal, Redalyc, and Google Scholar. The results indicated that B-SPG has a satisfactory accumulation of psychometric properties; Its application in a research context expands the notions of the functioning of Brazilian children's perceptive maturity and that B-SPG Bender correction system also corroborates the practice of psychologists working in the field of Psychology and Education.

Keywords: perceptomotor maturity; child psychoeducational assessment; clinical practice; cognitive development; intervention.

\section{TEST DE BENDER - SISTEMA DE PUNTUACIÓN GRADUAL: SCOPING REVIEW}

\section{RESUMEN}

La prueba de Bender evalúa la madurez perceptomotora de niños a través de las copias de dibujos. Actualmente en Brasil, el único sistema disponible para la corrección es el Sistema de Puntuación Gradual/B-SPG, que considera los errores de distorsiones del dibujo. Debido su contribución para la comprensión de las dificultades en la madurez perceptomotora presentada por niños y para la elaboración/acompañamiento de intervenciones, esta revisión (scoping review) presenta un panorama cerca del B-SPG (2005-2020). El analice cuantitativa y cualitativa fue con 35 artículos empíricos recuperados en el Portal de Periódicos CAPES, Redalyc y Google Scholar. Los resultados indicaran que el B-SPG tiene un acumulo satisfactorio de propiedades psicométricas; su aplicación en contexto de pesquisa amplia las nociones sobre el funcionamiento de la madurez perceptomotora de niños brasileños y que este sistema de corrección también corrobora la práctica de psicólogos actuantes en el campo de la Psicología y de la Educación.

Palabras clave: madurez perceptomotora; evaluación psicoeducativa infantil; práctica clínica; desarrollo cognitivo; intervención. 


\section{INTRODUÇÃO}

O teste Gestáltico Visomotor de Bender, ou teste de Bender como é comumente conhecido, é utilizado para avaliar a maturidade perceptomotora de crianças no âmbito da Psicologia Clínica e da Psicologia Escolar e Educacional (Bender, 1955; Lima, Cunha \& Suehiro, 2019; Keppeke \& Schoen, 2018; Santos, Anache \& Santana, 2015; Suehiro \& Cardim, 2016). A maturidade perceptomotora abarca as habilidades da criança de perceber e integrar os estímulos externos para expressálos em uma ação motora. Ao partir desta definição, o teste de Bender avalia a maturidade perceptomotora por meio da reprodução manual de figuras gestálticas. O modo como a criança executa as tarefas do teste de Bender permite compreender como ela se orienta dentro de um determinado quadro de referência e o seu modo de lidar com as relações espaciais (Bender, 1955; Koppitz, 1989; Sisto, Noronha \& Santos, 2006).

A pioneira em utilizar figuras gestálticas para analisar a percepção visual e a coordenação motora foi a neuropsiquiatra pediátrica estadunidense Lauretta Bender em 1955. O propósito da autora consistia em analisar apenas o refinamento dos desenhos, a fim de compreender se os erros cometidos na sua reprodução seriam provenientes de distúrbios cerebrais ou da imaturidade para reproduzir corretamente aquilo que foi percebido (Bender, 1955; Sisto et al., 2006).

Ao longo dos anos, os resultados obtidos com as pesquisas que utilizaram o teste de Bender demonstram a existência de relações entre a maturidade perceptomotora com diversas habilidades cognitivas como, por exemplo, a linguagem, os conceitos espaciais e temporais, a capacidade de organização, o planejamento, a atenção, a memória e a coordenação motora (Bender, 1955; Koppitz, 1989; Sisto et al., 2006). Os estudos que avaliaram crianças em idade escolar por meio do teste de Bender têm demonstrado que a maturidade perceptomotora, quando bem desenvolvida, associa-se positivamente com medidas que avaliam a inteligência, funções executivas e memória (Carreras, Uriel \& Liporace, 2013; Otoni \& Rueda, 2020); e com as habilidades ligadas ao rendimento escolar, como é o caso da escrita, da compreensão de leitura (Otoni $\&$ Rueda, 2019a; Soto \& Cruz, 2018) e da matemática (Betancur, Miranda, Valdivia \& Santisteban, 2017). Ademais, parece existir uma associação positiva entre as dificuldades na maturidade perceptomotora e de aprendizagem. Isto porque a capacidade de reproduzir manualmente estímulos visuais está presente na aquisição de novos conhecimentos e por ser uma habilidade primitiva que, portanto, segue um caráter desenvolvimental cognitivo, sugerindo que conforme a criança se desenvolve, melhor é a capacidade de percepção visual e integração dos estímulos (Soto, 2014; Soto, Allen \& Decker, 2016; Soto, Cruz \& Medina, 2016).

Inicialmente, o teste de Bender detinha apenas um enfoque clínico e qualitativo, visto que Bender (1955) não havia apresentado um método sistemático de correção e avaliação das figuras. Este fato gerou diversas críticas ao teste, dentre elas a falta de normatização para nortear a interpretação dos resultados, o que 
poderia prejudicar a credibilidade da avaliação feita com o instrumento (Bandeira \& Hutz, 1994; Pinelli Junior \& Pasquali, 1992; Sisto et al., 2006). Mediante essas críticas, diversos pesquisadores elaboraram diferentes métodos de correção e interpretação para as figuras do teste de Bender aplicados em crianças (foco principal do presente estudo), descritos a seguir em ordem cronológica.

O sistema de correção de Clawson (1959), utilizou o teste de Bender como método projetivo, o que permitida a análise de possíveis dificuldades emocionais da criança. Por sua vez, Santucci e Galifret-Granjon (1968) elaboraram um sistema de correção para o teste de Bender com o objetivo de diferenciar o nível de desenvolvimento de crianças de seis a 14 anos. A partir deste sistema, era possível identificar, além das falhas na organização perceptual e motora, outros aspectos como as dificuldades de aprendizagem e as oligofrenias (ex. problemas de interação com o mundo exterior) (Santucci \& Galifret-Granjon, 1968). Posteriormente, Santucci e Pêcheux (1981), criaram um sistema de correção para discriminar as crianças que possuíam dificuldades de aprendizagem das crianças com deficiência mental e, também, para detectar possíveis discordâncias entre o nível mental e o nível de organização espacial. Mais adiante, o sistema de pontuação proposto por Koppitz (1989) apresentava uma normatização de correção, considerando além da maturidade perceptomotora, o caráter evolutivo do teste, bem como a investigação de aspectos ligados ao desempenho escolar, de cunho emocional e diagnósticos de lesão cerebral. Mais à frente, Brannigan e Brunner (2002) elaboraram o Teste Gestáltico de Bender Modificado (TGBM), centralizado na análise da representação global de cada desenho, com pontuação variando de zero a cinco pontos, sendo sensível para captar as diferenças na configuração gestáltica dos traçados. Já em 2006, foi proposto o teste Gestáltico Visomotor de Bender - Sistema de Pontuação Gradual (B-SPG) por Sisto et al. (2006). Essa foi a primeira iniciativa de compor um sistema de correção para o teste de Bender voltado para a realidade brasileira e configura-se o foco principal do presente estudo de revisão.

O B-SPG foi pensado a partir de inúmeras críticas de pesquisadores brasileiros e estrangeiros direcionadas ao método de pontuação de Koppitz. A exemplo disto, se destaca a falta de evidências de validade deste sistema de pontuação para a predição do rendimento escolar, suas limitações para a elaboração de diagnósticos neuropsicológicos, a identificação de divergências entre a captação da faixa etária de cada criança, diferenças entre os sexos e a inapropriação para administração em crianças de 10 anos, o que se configura em ausência de evidência de validade de critério concorrente (La Puente \& Maciel Junior, 1984; Pinelli \& Pasquali, 1992; Ungaretti \& Bonamigo, 1985). No Brasil, além de encontrarem fragilidades em relação aos critérios de correção propostos pela autora (Sisto, Noronha \& Santos, 2004), o sistema de Koppitz não apresentou evidências de validade preditiva para as dificuldades de aprendizagem (Bartholomeu, Rueda \& Sisto, 2005) e de critério concorrente na diferenciação do desempenho em razão da idade (Sisto, Santos \& Noronha, 2004). 
Apoiados nas considerações supracitadas e nos pressupostos de Bender (1955), Sisto et al. (2006) criaram um sistema de pontuação direcionado para crianças. Este sistema apresentava novas normas para a aplicação individual e coletiva do teste de Bender, e manteve o caráter evolutivo da maturidade perceptomotora. O B-SPG compreende a maturidade perceptomotora por meio de padrões de diferentes graus de complexidade e princípios de organização. Neste sistema de pontuação, o teste de Bender se constitui de nove figuras. A pontuação é gradual, variando de zero a três pontos - pontuação máxima de 21 pontos. O B-SPG considera somente os erros de distorção da forma, assim, quanto maior a pontuação, menor é a precisão na reprodução dos aspectos estruturais da figura (Sisto et al., 2006).

A proposta de normatização do B-SPG foi feita por Sisto et al. (2006), a partir de uma amostra de 1052 crianças matriculadas entre a pré-escola e a $4^{a}$ série (atual $1^{\circ}$ e $5^{\circ}$ ano) do Ensino Fundamental I que responderam o teste de Bender em aplicações coletivas. Com base no modelo Rasch, as autoras do estudo identificaram até três níveis de distorção em cada uma das figuras do teste e uma sequência hierárquica do nível de dificuldade de reprodução, a saber, alto nível de dificuldade, Figuras 2, 4 e 3; nível médio, Figuras 1, 6, 7a e 7b; e baixo nível de dificuldade, Figuras A, 5 e 8. Atualmente, o B-SPG é o único sistema de correção e interpretação para o teste de Bender com parecer favorável pelo Sistema de Avaliação de Testes Psicológicos (Conselho Federal de Psicologia, 2001, 2003) no Brasil para fins diagnósticos.

Ao focalizar a utilização do B-SPG por profissionais da Psicologia, sobretudo na clínica e nas escolas, é de extrema importância que este sistema de correção detenha propriedades psicométricas adequadas e compatíveis com as especificidades do público-alvo avaliado e ao contexto que será aplicado. Nesse sentido, aponta-se para as estimativas de fidedignidade, com a finalidade de garantir a acurácia deste sistema, e a apresentação de evidências de validade, imprescindíveis para a sustentação das interpretações dos resultados obtidos com o teste de Bender (Americam Educational Research Association [AERA], American Psychological Association [APA] \& National Conuncilon Measurement in Education [NCME], 2014). Mediante esses pré-requisitos, a presente revisão teve por objetivo verificar a qualidade psicométrica do teste de Bender por meio da produção científica com o B-SPG, com ênfase para as estimativas de fidedignidade e as evidências de validade apresentadas. O presente estudo de revisão parte da concepção de que os instrumentos destinados à avaliação de construtos psicológicos são importantes ferramentas para informar o nível de desempenho do sujeito avaliado (nesse caso crianças em idade escolar) e na elaboração, revisão e acompanhamento de intervenções com o intuito de estimular/desenvolver a maturidade perceptomotora. Destarte, focalizar o sistema de correção e pontuação de um instrumento amplamente utilizado, como é o caso do teste de Bender, contribui tanto para a ampliação e atualização do conhecimento científico a respeito do que tem sido produzido, como para divulgar os potenciais benefícios da sua aplicação, uma vez que o psicólogo terá acesso aos contextos e casos em que o B-SPG tem sido mais relevante. 


\section{MÉTODO}

O presente estudo de revisão enquadra-se na modalidade do scoping review. Este tipo de revisão pressupõe a apresentação de um panorama geral sobre a produção científica acerca de um determinado assunto. O scoping review pode ser replicado, por apresentar, por exemplo, aspectos metodológicos relativos à divulgação da estratégia de busca utilizada, os critérios de elegibilidade, a seleção e os procedimentos de coleta das informações dos estudos recuperados (Grant \& Booth, 2009).

\section{ESTRATÉGIAS DE BUSCA}

As buscas dos estudos foram realizadas no Portal de Periódicos CAPES, na Red de Revistas Científicas de América Latina y el Caribe, España y Portugal (Redalyc) e no Google Scholar. Como o objetivo da presente revisão era abarcar apenas as pesquisas com o B-SPG, primeiramente realizou-se uma consulta prévia de estudos sobre o uso do teste de Bender e, por conseguinte, delimitou-se para a execução das buscas o período de janeiro de 2005 (ano anterior de publicação do manual do B-SPG) a maio de 2021. As palavras-chave utilizadas na recuperação dos estudos foram "teste de Bender" AND "Bender - Sistema de Pontuação Gradual" OR "B-SPG" e suas respectivas versões para o inglês e o espanhol.

\section{CRITÉRIOS DE ELEGIBILIDADE DOS ESTUDOS}

Os critérios de inclusão dos estudos recuperados pressupunham a correção e pontuação do teste de Bender por meio do B-SPG; a avaliação da maturidade perceptomotora centralizada nos aspectos cognitivos; pesquisas empíricas e revisadas por pares. Em vias de atender esses critérios de elegibilidade, foram excluídas as pesquisas que aplicaram somente outro sistema de correção/pontuação para o teste de Bender; estudos com o propósito de avaliar aspectos socioemocionais a partir da avaliação da maturidade perceptomotora; artigos teóricos e de revisão; trabalhos de monografia; dissertação de mestrado e tese de doutorado.

\section{SELEÇÃO E PROCESSO DE COLETA DE DADOS}

Após a recuperação dos artigos, foram lidos os seus respectivos títulos e resumos. A partir desta leitura prévia foram excluídos os artigos que não atendiam os critérios de elegibilidade. Os artigos que permaneceram foram lidos na íntegra para verificar se faziam parte do escopo. Posteriormente, foram extraídas as informações dos artigos que se enquadraram no escopo da revisão, com base nos seguintes descritores: autoria, ano, características da amostra - quantidade, sexo, idade, país de origem, escolaridade, tipo de escola (pública ou privada), os objetivo de cada estudo, as propriedades psicométricas investigadas com o B-SPG, demais instrumentos relacionados ao teste de Bender-SPG, os avanços científicos sobre a maturidade perceptomotora obtidos com esses estudos, questões a serem 
investigadas em futuros estudos com o B-SPG e, por fim, a contribuição deste instrumento/sistema de correção para a prática do profissional da Psicologia.

\section{RESULTADOS E DISCUSSÃO}

Por meio das buscas foram recuperados 794 estudos. Dentre eles, 759 estudos foram removidos por serem duplicados e/ou por estarem fora do escopo da presente revisão. Ao final, permaneceram 35 artigos (Figura 1), que foram avaliados por meio de análises de frequência e qualitativa.
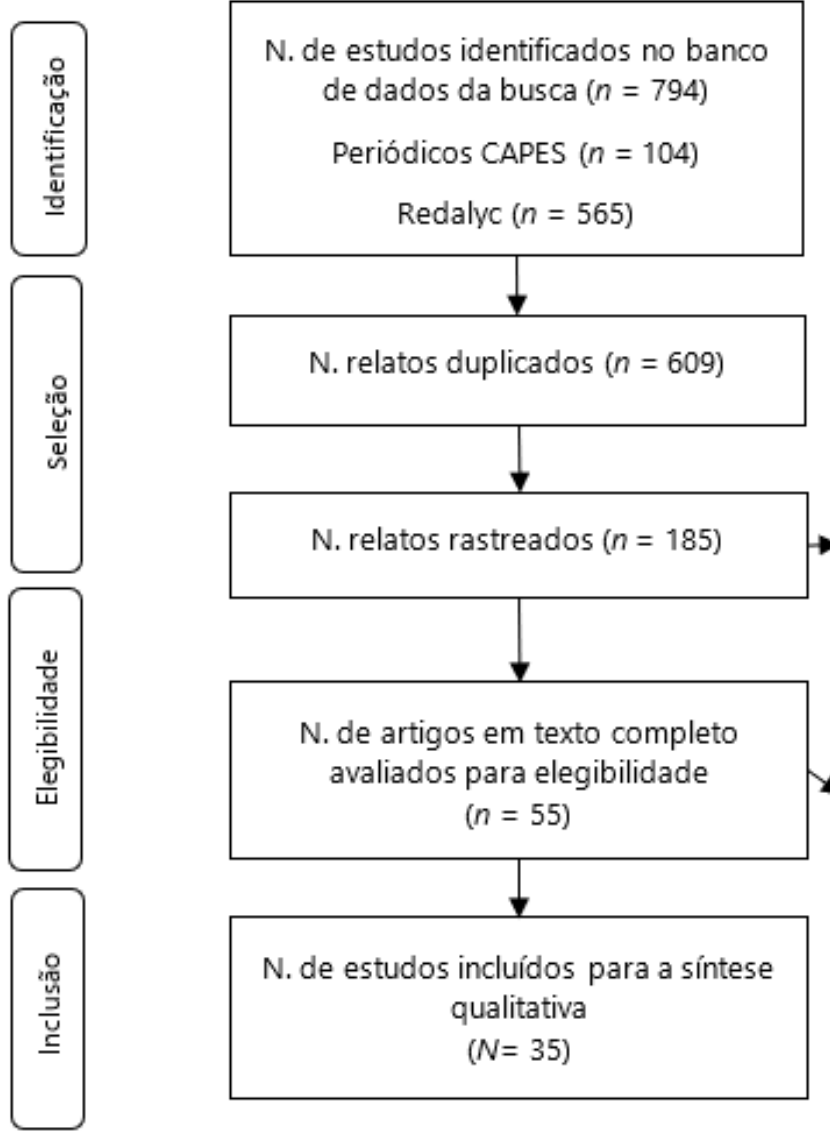

N. relatos excluídos $(n=130)$

N. de artigos excluídos com justificativa

Artigo teórico $(n=5)$

Artigo de revisão $(n=3)$

Dissertação $(n=2)$

Tese $(n=5)$

Figura 1. Fluxograma dos artigos encontrados nos bancos de dados.

Para atender os objetivos propostos, primeiramente, os estudos recuperados pela estratégia de busca foram organizados em uma tabela considerando as características da amostra (Tabela 1). Em seguida, elaborou-se três tópicos temáticos para discutir as propriedades psicométricas dos estudos realizados com o B-SPG; os avanços científicos e futuras pesquisas; e o uso do instrumento na prática profissional da Psicologia.

Os resultados indicaram que as publicações com o B-SPG datam o período de 2005 a 2020, sendo os anos de $2008(n=5 ; 15 \%)$ e $2016(n=4 ; 13 \%)$ com maior predominância de artigos. Apenas o ano de 2009 não apresentou estudos com o sistema de correção do teste de Bender. O tamanho amostral variou de 20 a 1.148 
participantes, com idades entre seis a 30 anos de idade, e escolaridade entre o primeiro ao nono ano, de escolas públicas e particulares do Ensino Fundamental. Observou-se que em grande parte das pesquisas, as crianças apresentavam desenvolvimento típico, no entanto, alguns estudos relataram usar parte da amostra com crianças que apresentavam dificuldades de aprendizagem (ex. dislexia), diagnóstico de Síndrome de Down, transtorno do desenvolvimento intelectual e não abrigadas. Além disso, foram realizadas pesquisas com crianças peruanas e de oito estados brasileiros, que contemplam as regiões do Sudeste, Nordeste, Centro-Oeste e Sul. Os resultados que descrevem as características dos estudos e das amostras avaliadas podem ser visualizados na Tabela 1.

Tabela 1.

Características descritivas da amostra e dos estudos.

\begin{tabular}{|c|c|c|c|c|}
\hline Autoria/Ano & Ano & $\mathbf{N}$ amostral & Sexo & Idade \\
\hline Suehiro e Santos & 2005 & 287 & $\begin{array}{l}134 \text { meninas } \\
153 \text { meninos }\end{array}$ & 7 e 10 \\
\hline $\begin{array}{l}\text { Noronha e } \\
\text { Mattos }\end{array}$ & 2006 & 85 & $\begin{array}{l}18 \text { meninas } \\
67 \text { meninos }\end{array}$ & 6 e 10 \\
\hline Suehiro e Santos & 2006 & 237 & $\begin{array}{l}114 \text { meninas } \\
123 \text { meninos }\end{array}$ & 8 e 9 \\
\hline $\begin{array}{c}\text { Noronha, Santos } \\
\text { e Sisto }\end{array}$ & 2007 & 1052 & $\begin{array}{l}527 \text { meninas } \\
525 \text { meninos }\end{array}$ & 6 e 10 \\
\hline Santos e Jorge & 2007 & 20 disléxicos & $\begin{array}{l}4 \text { meninas } \\
16 \text { meninos }\end{array}$ & 9 e 16 \\
\hline $\begin{array}{c}\text { Suehiro, Rueda e } \\
\text { Silva }\end{array}$ & 2007 & $\begin{array}{c}128-64 \\
\text { abrigadas }\end{array}$ & $\begin{array}{l}60 \text { meninas } \\
68 \text { meninos }\end{array}$ & 7 e 10 \\
\hline $\begin{array}{l}\text { Bartholomeu e } \\
\text { Sisto }\end{array}$ & 2008 & 244 & $\begin{array}{l}122 \text { meninas } \\
122 \text { meninos }\end{array}$ & 7 e 10 \\
\hline $\begin{array}{c}\text { Pacanaro, Santos } \\
\text { e Suehiro }\end{array}$ & 2008 & $\begin{array}{l}51 \text { - Síndrome } \\
\text { de Down }\end{array}$ & $\begin{array}{l}26 \text { meninas } \\
25 \text { meninos }\end{array}$ & 6 e 24 \\
\hline $\begin{array}{l}\text { Rueda, Suehiro e } \\
\text { Silva }\end{array}$ & 2008 & 80 & $\begin{array}{l}38 \text { meninas } \\
42 \text { meninos }\end{array}$ & 6 e 11 \\
\hline $\begin{array}{c}\text { Sisto, } \\
\text { Bartholomeu, } \\
\text { Rueda, Santos e } \\
\text { Noronha }\end{array}$ & 2008 & 239 & $\begin{array}{l}117 \text { meninas } \\
122 \text { meninos }\end{array}$ & 7 e 10 \\
\hline $\begin{array}{l}\text { Vendemiatto, } \\
\text { Santos e Suehiro }\end{array}$ & 2008 & 41 & $\begin{array}{l}22 \text { meninas } \\
19 \text { meninos }\end{array}$ & 13 e 17 \\
\hline
\end{tabular}




\begin{tabular}{|c|c|c|c|c|}
\hline $\begin{array}{l}\text { Lima, Cunha, } \\
\text { Santos e Mognon }\end{array}$ & 2010 & 148 & $\begin{array}{l}80 \text { meninas } \\
68 \text { meninos }\end{array}$ & 7 e 13 \\
\hline Pinto e Noronha & 2010 & 298 & $\begin{array}{l}143 \text { meninas } \\
155 \text { meninos }\end{array}$ & 6 e 10 \\
\hline $\begin{array}{l}\text { Sisto, Santos e } \\
\text { Noronha }\end{array}$ & 2010 & 1052 & $\begin{array}{l}539 \text { meninas } \\
513 \text { meninos }\end{array}$ & 6 e 10 \\
\hline Soto & 2011 & 32 & $\begin{array}{l}19 \text { meninas } \\
12 \text { meninos }\end{array}$ & 5 e 6 \\
\hline $\begin{array}{l}\text { Bartholomeu, } \\
\text { Cecato, Montiel, } \\
\text { Machado e Sisto }\end{array}$ & 2012 & 112 & $\begin{array}{l}57 \text { meninas } \\
55 \text { meninos }\end{array}$ & 7 e 9 \\
\hline $\begin{array}{c}\text { Carvalho, } \\
\text { Noronha, Pinto e } \\
\text { Luca }\end{array}$ & 2012 & 297 & $\begin{array}{l}144 \text { meninas } \\
153 \text { meninos }\end{array}$ & 7 e 10 \\
\hline $\begin{array}{c}\text { Rueda, Santos, } \\
\text { Noronha e } \\
\text { Segovia }\end{array}$ & 2012 & 1134 & $\begin{array}{l}311 \text { meninas } \\
823 \text { meninos }\end{array}$ & 8 e 10 \\
\hline $\begin{array}{c}\text { Noronha, Santos } \\
\text { e Rueda }\end{array}$ & 2013 & $\begin{array}{c}397 \\
\text { deficientes } \\
\text { intelectuais }\end{array}$ & $\begin{array}{l}157 \text { mulheres } \\
240 \text { homens }\end{array}$ & 6 e 24 \\
\hline $\begin{array}{l}\text { Noronha, Rueda } \\
\text { e Santos }\end{array}$ & 2013 & 511 & $\begin{array}{l}255 \text { meninas } \\
256 \text { meninos }\end{array}$ & 6 e 10 \\
\hline Pinto e Noronha & 2013 & 361 & $\begin{array}{l}193 \text { meninas } \\
168 \text { meninos }\end{array}$ & 6 e 10 \\
\hline $\begin{array}{l}\text { Santos, Noronha, } \\
\text { Rueda e Segovia }\end{array}$ & 2014 & 231 & $\begin{array}{l}105 \text { meninas } \\
126 \text { meninos }\end{array}$ & 6 e 10 \\
\hline $\begin{array}{c}\text { Noronha, Rueda } \\
\text { e Santos }\end{array}$ & 2015 & 1148 & $\begin{array}{l}563 \text { meninas } \\
585 \text { meninos }\end{array}$ & 6 e 10 \\
\hline $\begin{array}{c}\text { Suehiro, Santos e } \\
\text { Rueda }\end{array}$ & 2015 & 199 & $\begin{array}{l}93 \text { meninas } \\
106 \text { meninos }\end{array}$ & 7 e 10 \\
\hline $\begin{array}{l}\text { Batista e } \\
\text { Gonçalves }\end{array}$ & 2016 & 98 & $\begin{array}{l}48 \text { meninas } \\
50 \text { meninos }\end{array}$ & 9 e 10 \\
\hline Oliveira et al. & 2016 & 83 & $\begin{array}{l}50 \text { meninas } \\
33 \text { meninos }\end{array}$ & 7 e 10 \\
\hline $\begin{array}{l}\text { Rueda, Sousa, } \\
\text { Santos e } \\
\text { Noronha }\end{array}$ & 2016 & 787 & $\begin{array}{l}405 \text { meninas } \\
382 \text { meninos }\end{array}$ & 6 e 10 \\
\hline Suehiro e Cardim & 2016 & 388 & 202 meninas & 7 e 10 \\
\hline
\end{tabular}


186 meninos

\begin{tabular}{|c|c|c|c|c|}
\hline Sousa e Rueda & 2017 & 320 & $\begin{array}{l}196 \text { meninas } \\
124 \text { meninos }\end{array}$ & 6 e 10 \\
\hline $\begin{array}{c}\text { Silva, Oliveira e } \\
\text { Ciasca }\end{array}$ & 2017 & 24 & $\begin{array}{l}9 \text { meninas } \\
15 \text { meninos }\end{array}$ & 7 e 9 \\
\hline Valderas et al. & 2017 & 108 & $\begin{array}{l}\text { meninas } \\
\text { meninos }\end{array}$ & 6 e 11 \\
\hline Rueda e Jesuíno & 2018 & 401 & $\begin{array}{l}209 \text { meninas } \\
192 \text { meninos }\end{array}$ & 7 e 30 \\
\hline Otoni e Rueda & $2019 a$ & 333 & $\begin{array}{l}179 \text { meninas } \\
154 \text { meninos }\end{array}$ & 6 e 10 \\
\hline Otoni e Rueda & $2019 b$ & 693 & $\begin{array}{l}365 \text { meninas } \\
328 \text { meninos }\end{array}$ & 6 e 10 \\
\hline Otoni e Rueda & 2020 & 693 & $\begin{array}{l}365 \text { meninas } \\
328 \text { meninos }\end{array}$ & 6 e 10 \\
\hline
\end{tabular}

\section{ESTUDOS PSICOMÉTRICOS REALIZADOS COM O B-SPG}

Parte dos estudos que buscaram por evidências de validade com base na estrutura interna compararam o método de correção do B-SPG com o Sistema de Koppitz (Noronha \& Mattos, 2006), o Sistema de Classificação Global (Soto, 2011) e com o Método Qualitativo de Bender (Valderas et al., 2017). Com o objetivo de verificar se os escores obtidos a partir do B-SPG poderiam apresentar diferenças na interpretação dos resultados, a depender do método de correção utilizado, Noronha e Mattos (2006) realizaram a análise de correlação $r$ de Pearson e encontraram resultados significativos, cujos índices variaram de $r=0,69$ a $r=0,78$ e a magnitude foi de moderada a forte. Tendo em vista que todos os sistemas de correção mantêm o caráter evolutivo da maturidade perceptomotora, estes estudos ainda consideraram as idades dos participantes, além das correlações se manterem significativas, apresentaram magnitude moderada $(r=0,50$ a $r=0,61)$.

Rueda et al. (2008) buscaram por evidências de validade com base na estrutura interna por meio da concordância entre avaliadores, no qual as correlações estatisticamente significativas apresentaram uma magnitude forte $(r=0,70$ a $r=$ 0,92), tanto no teste como no re-teste. Os autores sugerem que os critérios de correção para cada uma das figuras do teste de Bender no sistema de pontuação gradual é claro e preciso, contribuindo para que a subjetividade do avaliador não interfira no escore da criança no teste.

Ainda com base na estrutura interna, o B-SPG conta com estudos que utilizaram análises estatísticas mais robustas como a de Funcionamento Diferencial de Item (DIF) e a Análise Fatorial Exploratória (AFE). Com o propósito de verificar a 
possibilidade de obter uma versão de rastreio da maturidade perceptomotora com um menor número de figuras, Rueda et al. (2016) realizaram uma AFE. O resultado indicou que as figuras 3, 4 e 7, são capazes de explicar aproximadamente $80 \%$ da maturidade perceptomora. Além disso, os autores selecionaram figuras que seguem os diferentes princípios da Gestalt, a saber, a proximidade, o fechamento e a integração, o que possibilita verificar os diferentes erros de distorção cometidos pelas crianças.

A análise de DIF, por sua vez, foi utilizada para verificar se alguma figura do B-SPG poderia favorecer o desempenho de meninas ou meninos (Sisto et al., 2010); ou de crianças brasileiras e peruanas (Santos et al., 2014); ou ainda, de pessoas com o desenvolvimento típico daquelas com diagnóstico diferencial (Rueda \& Jesuíno, 2018). Os resultados indicaram que a Figura 5 e a Figura 6 favorecem o desempenho em razão do sexo, sendo a primeira mais difícil para os meninos e a segunda mais fácil para as meninas. Já a Figura 1 e a Figura 4 apresentam diferenças quando considerada a nacionalidade das crianças, sendo esta última mais fácil para as crianças peruanas acertarem enquanto as crianças brasileiras têm maior facilidade na reprodução da Figura 1. Sisto et al. (2010) e Santos et al. (2014) consideraram que as figuras se sobrepõem em níveis de dificuldade, não sendo necessária a elaboração de tabelas normativas separadas. Rueda e Jesuíno (2018) verificaram que crianças sem comprometimento intelectual têm maior facilidade na reprodução da Figura 6, enquanto o outro grupo é mais favorecido pela Figura 1 e a Figura 2. Esses resultados sugerem que crianças com desenvolvimento atípico frequentam escolas que atendem demandas específicas, ligadas às necessidades especiais, e por isso, podem ser mais estimuladas em relação a percepção visual e a coordenação motora. Todavia, esses achados alertam sobre o cuidado para a produção de estudos que investiguem além do DIF em diferentes grupos, quais são as possíveis intervenções que podem ser feitas para favorecer as atividades que envolvem a maturidade perceptomotora.

Por reconhecer que o teste de Bender pressupõe o caráter desenvolvimental da maturidade perceptomotora, diversos estudos tiveram o intuito de verificar se o BSPG é um sistema de correção capaz de diferenciar o desempenho das crianças em função do avanço da idade e da escolaridade (Noronha et al., 2007; Pinto \& Noronha, 2010, 2013; Suehiro \& Cardim, 2016; Suehiro \& Santos, 2006). Por meio de análises de variância (Anova) observou-se que havia diferenças estatisticamente significativas ( $p<0,005$ ) entre e dentre os grupos, nas provas post hoc de Tukey, além de indicar quais os grupos que se diferenciaram, demonstrando que conforme o avanço da idade e dos anos escolares, menores são os erros de distorção de forma cometidos pelas crianças na reprodução das figuras do Bender. Pondera-se que os estudos que foram feitos com adolescentes e jovens adultos não apresentaram resultados tão favoráveis que diferenciassem as idades e a escolaridade dos participantes (Lima et al., 2010; Pacanaro et al., 2008; Vendemiatto et al., 2008). Os estudos que buscaram verificar possíveis diferenças na maturidade perceptomotora em razão do tipo de escola, particular ou pública, não encontraram resultados estatisticamente significativos. 
Em relação às diferenças de média de desempenho em razão do sexo, foram realizadas investigações apenas utilizando a análise do teste t de Student, no qual não foi possível chegar a uma conclusão se um determinado grupo de crianças tende a se sair melhor do que o outro (Batista \& Gonçalves, 2016; Pinto \& Noronha, 2010; Suehiro \& Cardin, 2016). A partir das mesmas análises, Rueda et al. (2012) utilizaram a amostra normativa do manual do B-SPG com crianças peruanas e não encontraram diferenças de médias estatisticamente significativa entre os grupos. Em outro estudo semelhante com crianças mineiras e paraibanas, Noronha et al. (2015) também não encontraram estas diferenças entre os grupos. Noronha et al. (2013), por sua vez, aplicaram a análise de variância (Anova) e não encontraram diferenças estatisticamente significativas entre crianças do estado de Minas Gerais, da Paraíba, do Piauí e de São Paulo. Estes resultados sugerem que o contexto regional/cultural tende a não interferir em relação à maturidade perceptomotora infantil, no entanto, aponta-se para a necessidade de desenvolver novos estudos com outros estados que ainda não foram contemplados.

Evidências de validade na relação com critério também foram feitas com crianças que apresentavam dificuldades de aprendizagem (Suehiro \& Santos, 2005), queixas de rendimento escolar (Silva et al., 2017) e baixo desempenho cognitivo (Bartholomeu et al., 2012). De modo geral, evidenciou-se que esses grupos apresentaram uma tendência em terem maiores distorções da reprodução das figuras do teste. Além disso, Santos e Jorge (2007) observaram que o B-SPG é um instrumento capaz de diferenciar o desempenho em crianças disléxicas. Pacanaro et al. (2008), por sua vez, verificaram que pessoas com Síndrome de Down tendem a apresentar um déficit na maturidade perceptomotora. Outro estudo realizado por Suehiro et al. (2017) foi verificar se mudanças ambientais poderiam influenciar no desempenho de crianças abrigada e não abrigadas. Embora crianças que moravam junto com os pais tenham apresentado menores erros de distorção na reprodução das figuras do B-SPG, os resultados não apontaram para diferenças estatisticamente significativas.

Ao considerar que a maturidade perceptomotora abarca habilidades cognitivas subjacentes à inteligência, o B-SPG foi correlacionado com os testes Desenho da Figura Humana-Escala Sisto (Bartholomeu \& Sisto, 2008), as Matrizes Progressivas Coloridas de Raven (Noronha et al., 2013; Sisto et al., 2008) e o Teste de Silver (Batista \& Gonçalves, 2016). A correlação ( $r$ de Pearson) entre esses instrumentos foi estatisticamente significativa, negativa e de magnitude moderada, cujos coeficientes variaram de $r=-0,49$ a $r=-0,60$, indicando que quanto menores são os erros de distorção no B-SPG maiores são as chances de as crianças pontuarem mais alto em testes de inteligência não verbal. Em outro estudo similar, Vendemiatto et al. (2008) correlacionaram o B-SPG com o teste de inteligência não verbal (R1-Forma $B$ ) e não encontraram resultados estatisticamente significativos, entretanto, a amostra utilizada por esses autores foi de 13 a 17 anos. $O$ fato de o B-SPG ser um sistema criado para o público de seis a 10 anos de idade pode ter contribuído para este resultado, sugerindo que o teste não é suficientemente 
preciso para avaliar a maturidade perceptomotora de sujeitos que extrapolam essa faixa etária.

Ainda sobre as evidências de validade baseadas nas variáveis externas, foram analisadas as correlações entre o desempenho do B-SPG com as Figuras Complexas de Rey (Oliveira et al., 2017) e a Bateria Psicológica para Avaliação da Atenção - BPA (Sousa \& Rueda, 2017). Os autores verificaram que quanto melhor é a reprodução das figuras do Bender, maior é a tendência de pontuar mais alto em tarefas que avaliam o planejamento, a memória de trabalho e a atenção. Carvalho et al. (2012) e Suehiro et al. (2015) tiveram o objetivo de verificar o desempenho no B-SPG com instrumentos que avaliam a compreensão de leitura e escrita (Teste de Reconhecimento de Palavras), e somente a escrita (Escala de Avaliação da Escrita [EAVE]). Os resultados apontaram para correlações estatisticamente significativas, negativas e de magnitude moderada, indicando que o desempenho em um instrumento interfere no desempenho do outro. Finalmente, Lima et al. (2010) verificaram que existe uma correlação estatisticamente significativa e de magnitude fraca entre a maturidade perceptomotora e o uso de jogos eletrônicos.

De modo geral, os estudos realizados com o B-SPG apresentaram um número considerável de evidências de validade, principalmente no que se refere à evidência de validade baseada em medidas que avaliam construtos relacionados. Conjecturase que esses estudos contribuem para as inferências que são feitas a partir dos escores das crianças no teste de Bender. Reconhece-se, também, que existem outros tipos de evidências de validade e estimativas de fidedignidade a serem investigados, que não foram contemplados nas pesquisas recuperadas e que poderiam favorecer a cobertura e a acurácia do B-SPG nos diversos contextos de avaliação psicológica. Dentre essas propriedades psicométricas, destaca-se a continuidade de estudos voltados à análise da consistência interna do B-SPG, o que permitiria verificar o quanto as figuras do teste de Bender estão livres de erros a depender da amostra avaliada; pesquisas que verifiquem as evidências de validade preditiva a fim de garantir que o B-SPG possa ser utilizado para predizer possíveis dificuldades de aprendizagem em longo prazo; e o uso de amostras clínicas, com crianças que apresentam diversos transtornos do desenvolvimento global.

Ao discorrer sobre a continuidade dos estudos psicométricos com o B-SPG, é preciso atentar-se para as estimativas de precisão ligadas as possíveis diferenças entre os avaliadores. Como é pressuposto para qualquer instrumento, é imprescindível que a psicóloga possua conhecimento sobre o teste de Bender antes de utilizá-lo. Segundo Rueda et al. (2012), diferenças nos resultados com este teste podem estar associadas ao nível de compreensão e da experiência da profissional da Psicologia sobre o B-SPG. Todavia, Sousa e Rueda (2017) reconhecem que existem disparidades, ainda que em grau reduzido, entre os resultados obtidos com o teste de Bender, e que estas diferenças podem estar 
associadas à subjetividade dos avaliadores, mesmo quando estes detêm prática com o método de correção e interpretação com o B-SPG.

Sobre a versão de rastreio do Bender, Rueda et al. (2016) reconhecem a necessidade de aplicar o seu conjunto de figuras isoladamente das demais figuras que compõem a versão do teste na íntegra. A ideia também é buscar pelas correlações existentes entre a versão de rastreio e outras variáveis e construtos subjacentes ao desempenho escolar. Muito disso já foi feito nas pesquisas de Otoni e Rueda $(2020,2019 b)$ que verificaram que o desempenho na versão de rastreio do B-SPG tende a influenciar em tarefas que dependem da inteligência, do planejamento e da memória, além de ser capaz de diferenciar o desempenho das crianças em razão da idade, mantendo o caráter maturacional da versão original do B-SPG. Ademais, os autores correlacionaram o instrumento com as notas escolares de crianças matriculadas no Ensino Fundamental I, observando que quanto maior a maturidade perceptomotora melhor é o desempenho nas disciplinas de português e matemática (Rueda \& Otoni, 2019a).

\section{AVANÇOS CIENTÍfICOS E TÓPICOS PARA A AGENDA DE FUTURAS PESQUISAS COM O B-SPG}

No tocante aos avanços na compreensão do funcionamento da maturidade perceptomotora em amostras brasileiras, destaca-se que níveis elevados desta habilidade estão associados às funções executivas, nomeadamente a percepção visual, a organização visual-espacial e a memória de trabalho (Oliveira et al., 2016). Outra característica da maturidade perceptomotora observada por Sousa e Rueda (2017) é a constante alteração do foco de atenção da criança entre a imagem observada e a cópia do desenho, fazendo com que as tarefas do teste de Bender estejam mais associadas à atenção alternada do que com a atenção concentrada.

As limitações identificadas na presente revisão também trazem diversas questões a serem melhor investigadas em futuras pesquisas com o B-SPG. Nesta perspectiva, conjectura-se a análise de uma provável interferência das habilidades artísticas da criança no nível do seu desempenho em maturidade perceptomotora. Isso porque esta característica pode impactar no bom desempenho da criança no teste, por minimizar as distorções na cópia dos desenhos independente do grau de maturidade perceptomotora (Bartholomeu et al., 2012; Bartholomeu \& Sisto, 2008).

Sobre a ampliação das amostras de estudo, sugere-se a comparação de alunos de escolas públicas e particulares a fim de conhecer o funcionamento da maturidade perceptomotora a partir de diferentes realidades/práticas pedagógicas e socioeconômicas, bem como a incidência de defasagem escolar (Suehiro \& Cardim, 2016). É indicada a continuidade de estudos que explorem a presença de diferenças no B-SPG quanto ao sexo, já que as pesquisas que abordaram esta variável têm identificado resultados heterogêneos (Pinto \& Noronha, 2010; Suehiro \& Cardim, 2016). Outro aspecto refere-se à verificação de possíveis diferenças socioculturais de crianças no teste de Bender ao considerar amostras de crianças abrigadas e não-abrigadas de uma mesma localidade, assim como de crianças e adolescentes 
em situação de risco (Suehiro et al., 2007; Vendemiatto et al., 2008). Uma vez que o B-SPG é capaz de diferenciar a maturidade perceptomotora em razão da idade, é proposta a investigação do nível de dificuldade das figuras do teste de Bender em razão desta variável, assim como a análise do limiar e das especificidades de cada grupo formado pelas faixas etárias e suas respectivas etapas desenvolvimentais (Noronha et al., 2007; Pinto \& Noronha, 2010, 2013).

Ainda é pouco explorado no Brasil estudos que abordam o desenvolvimento cognitivo de amostras atípicas a partir da maturidade perceptomotora (ex. sujeitos com deficiências físicas e intelectuais). É essencial em pesquisas dessa natureza, o cuidado com os aspectos metodológicos e éticos na sua condução para que não sejam cometidas injustiças com os sujeitos avaliados. Esses procedimentos englobam desde a seleção dos participantes, o tratamento dos dados, até a divulgação dos resultados. É primordial obter informações diagnósticas prévias que sejam fidedignas para a composição de amostras atípicas. Esses dados servem tanto para nortear os critérios de inclusão e exclusão como para verificar a prevalência de diferenças intergrupos (Rueda \& Jesuíno, 2018; Sousa \& Rueda, 2017). Essas precauções se somam à recomendação de Santos e Jorge (2007) sobre o tipo de aplicação do teste de Bender - individual ou coletiva. Estas autoras alertam para a possibilidade de existência de diferentes níveis de comprometimento no desenvolvimento nos grupos amostrais, o que podem interferir na graduação do sistema de correção e interpretação do B-SPG, tornando-o mais sensível às diferenças individuais.

No caso de sujeitos com deficiência intelectual, são necessários mais estudos para verificar se a maturidade perceptomotora destas pessoas se assemelha, de fato, com a de crianças de seis anos ou menos, em vias de adequar a normatização do B-SPG. Essa medida traria como benefício a utilização dos resultados do teste de Bender para a aplicação de intervenções em habilidade perceptomora com este público (Noronha et al., 2013). Neste mesmo seguimento, sabe-se que a maturidade perceptomotora de sujeitos com síndrome de Down tende a se desenvolver de forma mais lenta, além disso, parece haver diferenças nesta habilidade entre os sexos. À vista disto, recomenda-se a execução de estudos sobre o funcionamento diferencial das figuras do teste de Bender para esta população (Pacanaro et al., 2008). Outro modo de aperfeiçoar a medida para amostras de sujeitos com deficiência intelectual é o uso de curvas de informação dos itens do teste de Bender, com o propósito de melhorar as suas estimativas de precisão (Rueda \& Jesuíno, 2018).

Alguns estudos recuperados na presente revisão consideram pertinente explorar as possíveis particularidades do desempenho no teste de Bender em face das regiões brasileiras, pois os seus resultados ainda não convergem em um consenso sobre a existência de diferenças na maturidade perceptomotora quando se considera os aspectos regionais e culturais de cada localidade. A proposta de novas pesquisas se sustenta na necessidade de elaboração de tabelas normativas diferentes caso essas diferenças sejam identificadas (Noronha et al., 2013, 2015; 
Sousa \& Rueda, 2017). Este argumento também se aplica para outros países, como é o caso do Peru, caso venham a utilizar o B-SPG em contexto que não seja o de pesquisa (ex. para a formulação de diagnóstico clínico), o que implica na necessidade de desenvolver estudos psicométricos que abarquem a realidade contextual e do público-alvo o qual se destina (Rueda et al., 2012; Santos et al., 2014; Soto, 2011).

Ao conjecturar sobre as relações existentes entre a maturidade perceptomotora e a inteligência, Sisto et al. (2008) ponderam que a sua associação parece oscilar de fortemente correlacionada à independência do desenvolvimento de ambos os construtos, ainda que haja uma correlação entre eles. Desse modo, se faz pertinente a continuação de estudos com o objetivo de aprofundar nas suas relações, utilizando-se das várias perspectivas teóricas de inteligência e de diferentes tipos de medidas para avaliá-la (Sisto et al., 2008; Sisto et al., 2018). Outros aspectos a serem associados com a maturidade perceptomotora dizem respeito à leitura, escrita, aos problemas neuropsicológicos, as situações adversas de desenvolvimento infantil (Suehiro \& Cardim, 2016) e o uso de jogos eletrônicos (Lima et al., 2010). A este último também pode ser acrescida a utilização de smartphones, dado que estes aparelhos estão presentes no cotidiano de grande parte da população o qual se destina o teste de Bender.

\section{O USO DO B-SPg NA PRÁtICA PROFISSIONAL EM PSICOLOGIA}

O teste de Bender, sobretudo no sistema de pontuação gradual, corrobora a atuação da psicóloga na elaboração de diagnósticos clínicos e escolares por avaliar uma importante habilidade do desenvolvimento cognitivo infantil, bem como por ser um instrumento adequado à realidade das crianças e das escolas brasileiras (Noronha et al., 2013; Suehiro \& Santos, 2005). Conforme relatado ao longo da presente revisão, este sistema de correção apresenta inúmeros estudos de suas propriedades psicométricas (Valderas et al., 2017; Oliveira et al., 2016; Suehiro \& Cardim, 2016); possui baixos custos; pode ser aplicado coletivamente, por meio de projeções das figuras, o que reduz o tempo de avaliação (Rueda \& Jesuíno, 2018; Suehiro \& Santos, 2005); e dispõe de uma versão reduzida (de rastreio) que já apresenta estudos que reportam os indícios de sua potencial contribuição para a avaliação da maturidade perceptomotora (Rueda et al., 2016). Adicionalmente, conforme já apontavam Suehiro e Santos (2006), responder o teste de Bender também pode ser um atrativo para as crianças devido o seu caráter lúdico inerente à tarefa de copiar desenhos.

Considera-se vantajoso, por exemplo, utilizar o B-SPG na composição da avaliação cognitiva de sujeitos com alguma deficiência intelectual (ex. síndrome de Down), devido a simplicidade, tanto das orientações durante a sua aplicação como daquilo que é exigido em suas tarefas (Pacanaro et al., 2008). No contexto escolar, o teste de Bender pode ser utilizado em caráter preventivo, para identificar possíveis problemas de aprendizagem. No contexto clínico, conjectura-se que o uso da sua 
versão de rastreio pode otimizar o tempo das avaliações neuropsicológicas em casos que, logo no início, se observa a ausência de comprometimentos graves (Otoni \& Rueda, 2019a; Rueda et al., 2016).

Os resultados obtidos com o B-SPG trazem informações importantes para a psicóloga, uma vez que não considera apenas a presença ou ausência de erros nas tarefas, mas, também, a graduação dos erros de distorção da forma, fornecendo um panorama sobre o nível de comprometimento da maturidade perceptomotora da criança (Pinto \& Noronha, 2013; Santos \& Jorge, 2005). Assim, o sistema de pontuação gradual permite uma melhor interpretação acerca das dificuldades apresentadas. Essa característica do B-SPG é de grande importância quando se pensa na elaboração e no acompanhamento de intervenções feitas na clínica e/ou na escola (Santos \& Jorge, 2005; Suehiro \& Santos, 2005).

Ao adentrar nas práticas interventivas, é recomendado o uso do teste de Bender com sujeitos que possuem déficit intelectual e em casos que se identificam perdas nas habilidades perceptomotoras e na atenção (Noronha et al., 2013; Sousa \& Rueda, 2017). Ademais, devido a maturidade perceptomotora apresentar grande potencial para auxiliar no processo de ensino e aprendizagem, incentiva-se a disponibilização de atividades na escola com a finalidade de desenvolvê-las continuamente, acrescidas de outras habilidades, como as psicomotoras e intelectuais (Oliveira et al., 2016; Otoni \& Rueda, 2019b; Silva et al., 2017).

Indo mais além, é possível pensar na contribuição das pesquisas com o B-SPG para a formulação de políticas públicas nas áreas da Saúde e da Educação, as quais devem ser fundamentadas, dentre vários aspectos, em princípios científicos. A partir disso, as ações promovidas por essas políticas públicas apresentam maiores chances de permitirem à população as quais se destinam, meios de acesso a diagnósticos adequados e confiáveis, assim como encaminhamentos condizentes com a demanda apresentada. Esses cuidados pressupõem que a criança consiga superar suas dificuldades mediante a oferta de oportunidades de se desenvolver integralmente, considerando aqui os seus potenciais cognitivos (Vendemiatto et al., 2008).

\section{CONSIDERAÇÕES FINAIS}

Os estudos recuperados na presente revisão trazem importantes considerações que ampliam o conhecimento acerca do funcionamento da maturidade perceptomotora em amostras nacionais e estrangeiras de crianças em idade escolar e sujeitos com funcionamento atípico e com dificuldades de aprendizagem. Os bons índices psicométricos permitem inferir que o B-SPG é um instrumento fidedigno e válido, sendo indicado para a prática da psicóloga nos âmbitos da clínica e da escola. Todavia, aponta-se para a escassez de estudos de intervenção com o B-SPG. Ao considerar que a maturidade perceptomotora é uma habilidade inerente ao desenvolvimento humano, pesquisas com esse objetivo, principalmente com crianças que apresentam dificuldades de aprendizagem 
podem ampliar as possibilidades de uso do B-SPG com a finalidade de, por exemplo, contribuir para minimizar e, até mesmo, impedir comprometimentos intelectuais mais graves.

Nesse seguimento, sugere-se que em futuros estudos de revisão, sejam abordados outros instrumentos que apresentam como proposta a avaliação de habilidades cognitivas de crianças ainda no início da escolarização. Como exemplo pode-se mencionar o Desenho da Figura Humana - Escala Sisto, as Matrizes Progressivas Coloridas de Raven, a Escala Wechsler de Inteligência para Crianças - WISC-IV e o teste das Figuras Complexas de Rey. No que diz respeito ao presente estudo, identifica-se a necessidade de incluir em estudos de revisão a parcela emocional subjacente ao teste de Bender. Acredita-se que a maneira como a criança reproduz as figuras do teste pode dar indícios de aspectos emocionais das crianças. A partir de estudos que indiquem essa hipótese pode-se utilizar esses desenhos também para fazer uma análise qualitativa desses aspectos.

\section{REFERÊNCIAS}

American Educational Research Association, American Psychological Association, \& National Council on Measurement in Education, (2014). Standards for educational and psychological testing. Washington, DC: American Educational Research Association.

Bandeira, R. D. \& Hutz, C. S. (1994). A contribuição dos testes DFH, Bender e Raven na predição do rendimento escolar na primeira série. Psicologia: Teoria e Pesquisa, 10(1), 59-72.

*Batista, M. A., \& Gonçalves, V. A. (2016). Validade convergente entre os testes de Desenho de Silver (SDT) e Bender (B-SPG) por idade e sexo. Pecibes, 2(s/n), 44-51. Recuperado de http://www.seer.ufms.br/index.php/pecibes/index

*Bartholomeu, D., Cecato, J. F., Montiel, J. M., Machado, A. A., \& Sisto, F. F. (2012). Teste de Bender (BSPG) e DFH-Escala Sisto: validade por grupos contrastantes. Estudos Interdisciplinares em Psicologia, 3(2), 241-257. http://dx.doi.org/10.5433/2236-6407.2012v3n2p241

Bartholomeu, D., Rueda, F. J. M., \& Sisto, F. F. (2005). Teste de Bender e dificuldades de aprendizagem: quão válido é o sistema de Koppitz? Avaliação Psicológica, 4(1), 13-21. Recuperado de http://pepsic.bvsalud.org/pdf/avp/v4n1/v4n1a03.pdf

*Bartholomeu, D., \& Sisto, F. F. (2008). Maturidade viso-motora e inteligência: um estudo correlacional. Psicologia Ciência e Profissão, 28(2), 362-373. Recuperado de http://pepsic.bvsalud.org/scielo.php?script=sci_arttext\&pid=S141498932008000200011\&lng=pt\&tlng=pt

Bender, L. (1955). Teste Gestáltico Visomotor (Trad. D. Carnelli). Buenos Aires: Paidos.

Betancur, H. N. C., Miranda, P. S. Y., Valdivia, S. V., \& Santisteban, S. M. A. (2017). El test de Bender y las dificultades de aprendizaje en matemática de los estudiantes con necesidades especiales de la ciudad de Puno, Perú. Revista Electrónica Actualidades Investigativas en Educación 17(3), 1-16. https://doi.org/10.15517/aie.v17i3.29849

Brannigan, G. G., \& Brunner, N. A. (2002). Guide to the qualitative scoring system for the modified version of the Bender-Gestalt Test. Springfield: Publisher.

Carreras, M. A., Uriel, F., \& Liporace, M. F. (2013). Actualizaciones en el análisis de ítemes madurativos del dibujo de la figura humana en niños escolarizados de Buenos Aires. Interdisciplinaria, 30(1), 101-118. Recuperado de https://www.redalyc.org/pdf/180/18027808006.pdf

*Carvalho, L., Noronha, A. P. P., Pinto, L. P., \& Luca, L. (2012). Maturidade perceptomotora e reconhecimento de palavras: estudo correlacional entre o Bender-Sistema de Pontuação Gradual e o Teste de Reconhecimento de Palavras. Estudos de Psicologia (Campinas), 29(3),371-377 https://doi.org/10.1590/S0103-166X2012000300007

Conselho Federal de Psicologia (CFP) (2001). Resolução no 025/2001. [Online]. Recuperado de http://www.pol.org.br 
Conselho Federal de Psicologia. (2003). Resolução N 002/2003. [Online]. Define e regulamenta o uso, a elaboração e comercialização de testes psicológicos e revoga a resolução CFP n 025/2001. Recuperado de http://www.pol.org.br

Clawson, A. (1959). The Bender Visual Motor Gestalt Test as an index of emotional disturbance in children. Journal of Projective Techniques, 23(2), 198-206. https://doi.org/10.1080/08853126.1959.10380911

Grant, M. J., \& Booth, A. (2009). A typology of reviews: an analysis of 14 review types and associated methodologies. Health Information \& Libraries Journal, 26(2), 91-108. https://doi.org/10.1111/j.1471-1842.2009.00848.x

Keppeke, L. F., \& Schoen, T. H. (2018). Perceptual-Motor Maturity in Adolescence and the Tanner Stages: A Study with Bender-Gradual Scoring System. The Spanish Journal of Psychology, 21(33), 1-8. https://doi.org/10.1017/sjp.2018.33

Koppitz, E. M. (1989). O teste guestáltico Bender para crianças. Porto Alegre: Artes Médicas.

La Puente, M., \& Maciel Junior, J. (1984). Procedimentos operacionais na avaliação do Teste de Bender infantil. Estudos de Psicologia, 3(4), 76-92.

*Lima, T. H., Cunha, N. B., Santos, A. A. A., \& Mongon, J. F. (2010). Desenvolvimento visomotor e uso de jogos eletrônicos em crianças do ensino fundamental. Estudos Interdisciplinares em Psicologia, 1(2), 202-2015. http://dx.doi.org/10.5433/2236-6407.2010v1n2p202

Lima, T. H., Cunha, N. B., \& Suehiro, A. C. B. (2019). Produção Científica em avaliação psicológica no contexto escolar/educacional. Psicologia Escolar e Educacional, 23(3), 1-9. https://doi.org/10.1590/2175-35392019018897

*Noronha, A. P. P., \& Mattos, R. M. C. B. (2006). Koppitz e Bender-sistema de pontuação gradual: Comparação entre sistemas de avaliação. Psicologia Escolar e Educacional, 10(2), 223-233. Recuperado de http://www.redalyc.org/articulo.oa?id=282321819006

*Noronha, A. P. P., Rueda, F. J. M., \& Santos, A. A. A. (2015). Diferenças regionais e as normas de interpretação do Teste de Bender-Sistema de Pontuação Gradual. Psicologia em Pesquisa, 9(1), 3-9. http://dx.doi.org/10.5327/Z1982-1247201500010002

*Noronha, A. P. P., Santos, A. A. A., \& Sisto, F. F. (2007). Evidências de validade do Bender-sistema de pontuação gradual (B-SPG). Psicologia: Reflexão e Crítica, 20(2), 335-341. https://psycnet.apa.org/doi/10.1590/S0102-79722007000200020

*Noronha, A. P. P., Santos, A. A. A., \& Rueda, F. J. M. (2013). Habilidad viso-motriz y deficiencia intelectual: estudio de validez para el Bender-SPG. Acta Colombiana de Psicología, 16(2), 115 123. https://doi.org/10.14718/ACP.2013.16.2.11

*Noronha, A. P. P., Rueda, F. J. M., \& Santos, A. A. A. (2013). Teste Gestáltico Visomotor de Bender Sistema de Pontuação Gradual (B-SPG): A study with diferente samples. Paidéia (Ribeirão Preto), 23(55), 179-185. https://doi.org/10.1590/1982-43272355201305.

*Oliveira, A. L. S., Kaiser, V., Azambuja, T. O., Mallmann, L. U., Lukrafka, J. L., \& Reppold, C. T. (2016) Visual-motor maturity and executive functions in schoolchildren. Paidéia (Ribeirão Preto) 26(64), 215-223. https://doi.org/10.1590/1982-43272664201609

*Otoni, F., \& Rueda, F. J. M. (2020). Perceptive-Motor Maturity and its Relations with Planning, Memory Immediate and Non-Verbal Intelligence. Paidéia (Ribeirão Preto), 30, e3031. https://doi.org/10.1590/1982-4327e3031

*Otoni, F., \& Rueda, F. J. M. (2019a). Versão de rastreio do Teste de Bender para avaliar o desempenho escolar. Revista Psicologia: Teoria e Prática, 21(3), 28-44. http://dx.doi.org/10.5935/19806906/psicologia.v21n3p45-60

*Otoni, F., \& Rueda, F. J. M. (2019b). Teste de Bender: Versão de Rastreio para a Avaliação da Maturidade Perceptomotora. Avaliação Psicológica, 18(3), 316-324 http://dx.doi.org/10.15689/ap.2019.1803.16795.11

*Pacanaro, S. V., Santos, A. A. A., \& Suehiro, A. C. B. (2008). Avaliação das habilidades cognitiva e visomotora em pessoas com Síndrome de Down. Revista Brasileira de Educação Especial, 14(2) 311-326. https://doi.org/10.1590/S1413-65382008000200011

Pinelli Junior., B. \& Pasquali, L. (1992). Parâmetros para do teste Gestáltico Visomotor de Bender: um estudo empírico. Revista de Psicologia, 9(10), 51-74. Recuperado de file:///C:/Users/User/Downloads/1992_art_bpinellijunior.pdf

*Pinto, L. P., \& Noronha, A. P. P. (2010). Maturidade perceptomotora e sua relação com idade e variáveis contextuais: um estudo com o Bender (B-SPG). Encontro: Revista de Psicologia, $\begin{array}{lcr}\text { 13(19), } & \text { 145-155. Recuperado } \\ \text { http://www.pgsskroton.com.br/seer/index.php/renc/article/view/2522 }\end{array}$ 
*Pinto, L. P. \& Noronha, A. P. P. (2013). Bender-Sistema de Pontuação Gradual (B-SPG): Análise da Maturação Percepto-Motora de Crianças. Interação em Psicologia, 17(3), 281-289. http://dx.doi.org/10.5380/psi.v17i3.29683

*Rueda, F. J. M., \& Jesuíno, A. D. S. A. (2018). The Bender Gradual Scoring System in assessment of people with intellectual disabilities. Paideia (Ribeirão Preto), 28(8), 1-9. https://doi.org/10.1590/1982-4327e2807

${ }^{*}$ Rueda, F. M. J., Santos, A. A. A., Noronha, A. P. P., \& Segovia, J. L. (2012). Estudio transcultural com la prueba de Bender-sistema de pontuacióngradual. Liberabit, 19(2), 173-180. Recuperado de http://www.scielo.org.pe/scielo.php?script=sci_arttext\&pid=S1729$48272013000200003 \& \operatorname{lng}=$ es\&nrm =iso

*Rueda, F. J. M., Sousa, V., Santos, A. A. A., \& Noronha, A. P. P. (2016). Bender - Sistema de Pontuação Gradual (B-SPG): estudo para versão de rastreio. Psicologia: Teoria e Prática, 18(2), 117-128. http://dx.doi.org/10.15348/1980-6906/psicologia.v18n2p117-128

*Rueda, F. J. M., Suehiro, A. C. B., \& Silva, M. A. (2008). Precisão entre avaliadores e pelo método testereteste no Bender-Sistema de Pontuação Gradual. Psicologia: Teoria e Prática, 10(1), 25-35. Recuperado de http://pepsic.bvsalud.org/pdf/ptp/v10n1/v10n1a03.pdf

Santos, A. M., Anache, A. A., \& Santana, R. C. (2015). Overview of brazilian scientific production in psychological evaluation. Psico-USF, 20(3), 547-559. https://doi.org/10.1590/1413$\underline{82712015200315}$

*Santos, A. A. A., \& Jorge, L. M. (2007). Teste de Bender com disléxicos: comparação de dois sistemas de pontuação. Psico-USF, 12(1), 13-21. Recuperado de http://pepsic.bvsalud.org/scielo.php?script=sci arttext\&pid=S1413$82712007000100003 \&$ lng $=p t \& t \operatorname{lng}=p t$

*Santos, A. A. A., Noronha, A. P. P., Rueda, F. J. M., \& Segovia, J. L. (2014). Bender-Gradual scoring system: performance of Brazilian and Peruvian children. Perceptual and Motor Skills: Physical Development and Measurement, 118(3), 89-908. doi: 10.2466/03.10.PMS.118k25w7

Santucci, H., \& Galifred-Granjon, N. (1968). Prova gráfica de organização perceptiva. In R. Zazzo (Org.) Manual para o exame psicológico da criança (pp. 233-268). São Paulo: Editora Mestre Jou.

Santucci, H., \& Pêcheux, M. G. (1981). Prova gráfica de organização perceptiva para crianças de 6 a 14 anos. In R. Zazzo (Org.), Manual para o exame psicológico da criança (pp. 291-338). São Paulo: Editora Mestre Jou.

*Silva, S. L. Z. R., Oliveira, M. C. C., \& Ciasca, S. M. (2017). Desempenho percepto-motor, psicomotor e intelectual de escolares com queixa de dificuldade de aprendizagem. Revista Psicopedagogia, 34(103), 33-44. Recuperado de http://pepsic.bvsalud.org/scielo.php?script=sci_arttext\&pid=S0103-84862017000100004

*Sisto, F. F., Bartholomeu, D., Rueda, F. J. M., Santos, A. A. A., \& Noronha, A. P. P. (2008). Relações entre o teste de Bender e as Matrizes Progressivas Coloridas de Raven na avaliação da inteligência. Interação em Psicologia, 12(1), 11-19. Recuperado de https://www.researchgate.net/públication/273428450 Relacoes entre os Testes de Bende r e Matrizes Progressivas Coloridas de Raven na Avaliacao da Inteligência

Sisto, F. F., Noronha, A. P., \& Santos, A. A. A. (2004). Distorção da forma no teste de Bender: questionando seu critério de validade. Revista do Departamento de Psicologia da UFF, 16(2), 139-154

Recuperado

de

http://pepsic.bvsalud.org/scielo.php?script=sci_arttext\&pid $=$ S1677$04712004000100002 \&$ lng $=p t \& t \operatorname{lng}=p t$

Sisto, F. F., Noronha, A. P. P., \& Santos, A. A. A. (2006). Teste Gestáltico Visomotor de Bender: Sistema de pontuação gradual (B-SPG). Itatiba/SP: Vetor Editora Psicopedagógica Ltda.

Sisto, F. F., Santos, A. A. A., \& Noronha, A. P. (2004). Critério de Integração do Teste de Bender: Explorando Evidências de Validade. Avaliação Psicológica, 3(1), 13-20. Recuperado de http://pepsic.bvsalud.org/scielo.php?script=sci arttext\&pid $=\mathrm{S} 1677-04712004000100002$

*Sisto, F. F., Santos, A. A. A., \& Noronha, A. P. P. (2010). Diferential functioning of Bender visual-motor gestalts test items. Perceptual and Motor Skills, 110(1), 313-322. https://doi.org/10.2466\%2Fpms.110.1.313-322

*Soto, C. M. (2011). Exploración de diferencias normativas en el Sistema de Calificación Cualitativa para el Test Gestáltico de Bender Modificado. Liberabit, 17(2), 199-209. Disponível em: http://www.scielo.org.pe/scielo.php?script=sci arttext\&pid=S1729-48272011000200009 
Soto, C. M. (2014). Validez incremental del Test Gestáltico de Bender Modificado, en niños que inician el primer grado. Avances en Psicología Latinoamericana, 32(2), 275-286. http://dx.doi.org/10.12804/apl32.2.2014.07

Soto, C. M., Allen, R., \& Decker, S. (2016). Within-group inter-rater agreement for the Bender Gestalt visualmomor test, second version (Bender--II). Universitas Psychologica, 15(2), 163-171. http://dx.doi.org/10.11144/Javeriana.upsy15-2.aiit

Soto, C. M. \& Cruz, G. C. (2018). Validez de Constructo de una Prueba Breve de Pre-escrituraen Preescolares. Estudios pedagógicos (Valdivia), 44(3), 337-349. https://doi.org/10.4067/S071807052018000300337

Soto, C. M., Cruz, G. C., \& Medina, E. M. (2016). Estudio comparativo del acuerdo y consistencia intercalificadores en el test gestáltico visomotor de Bender 2.a edición. Revista Latinoamericana de Psicología, 48(3), 175-182. https://doi.org/10.1016/j.rlp.2015.09.011

*Sousa, V., \& Rueda, F. J. M. (2017). The relationship between perceptual motor skills and attention. Paidéia (Ribeirão Preto), 27(66), 24-32. https://doi.org/10.1590/1982 432727662017046201704

*Suehiro, A. C. B., \& Cardim, N. A. (2016). Bender-sistema de pontuação gradual: uma comparação por idade, ano e sexo em crianças baianas. Avaliação Psicológica, 15(2), 257-264. https://doi.org/10.15689/ap.2016.1502.14

*Suehiro, A. C. B., Rueda, F. J. M., \& Silva, M. A. (2007). Desenvolvimento perceptomotor em crianças abrigadas e não abrigadas. Paidéia (Ribeirão Preto), 17(38), 431-442. https://doi.org/10.1590/S0103-863X2007000300012

*Suehiro, A. C. B., \& Santos, A. A. A. (2005). O Bender e as dificuldades de aprendizagem: Estudo de validade. Avaliação Psicológica, 4(1), 23-31. Recuperado de http://www.redalyc.org/articulo.oa?id=335027178004

*Suehiro, A. C. B., \& Santos, A. A. A. (2006). Evidência de validade de critério do Bender-sistema de pontuação gradual. Interação em Psicologia, 10(2), 217-224. http://dx.doi.org/10.5380/psi.v10i2.7678

*Suehiro, A. C. B., Santos, A. A. A., \& Rueda, F. J. M. (2015). Desenvolvimento perceptomotor e escrita em crianças do ensino fundamental. Psicologia Escolar e Educacional, 19(2), 369-376. https://doi.org/10.1590/2175-3539/2015/0192861

Ungaretti, H. V., \& Bonamigo, E. M. R. (1985). O Teste de Bender como Preditor do Rendimento em Alfabetização. Prospectiva, 14(s/n), 52-60

*Valderas, R. V., Segovia, J. L., Moran, M. O., Alfaro, J. C., Vargas, G. S., Santos, A. A. A., Sisto, F. F., \& Noronha, A. P. P. (2017). La habilidad visomotora en niños escolares: un estúdio transcultural Perú-Brasil. PSIQUEMAG, 6(1), 73-89. Recuperado de http://ojs.ucvlima.edu.pe/index.php/psiquemag/article/view/174/92

*Vendemiatto, B. C., Santos, A. A. A., \& Suehiro, A. C. B. (2008). Inteligência e maturidade visomotora: estudo com adolescentes em situação de risco. Avaliação Psicológica, 7(3), 439-447. Recuperado de http://pepsic.bvsalud.org/scielo.php?script=sci arttext\&pid=S1677$\underline{04712008000300015 \& \mid n g=p t \& t \operatorname{lng}=p t}$

\section{CONFLITOS DE INTERESSES}

Declaramos que não há conflito de interesse de ordem pessoal, comercial, acadêmico, político e financeiro entre as partes.

\section{SOBRE OS AUTORES}

Fernanda Otoni é Mestre e doutoranda em Psicologia, com área de concentração em Avaliação Psicológica pelo Programa de Pós-Graduação Stricto Sensu da Universidade São Francisco. Membro do GT de Pesquisa em Avaliação Psicológica da Associação Nacional de Pesquisa e Pós-Graduação em Psicologia (ANPEPP). Atua, principalmente, em pesquisas psicométricas com construtos que envolvem o desenvolvimento infantil e habilidades cognitivas.

E-mail: fer_ottoni@hotmail.com

(2) https://orcid.org/0000-0002-9347-7144 
Adriana Satico Ferraz é Psicóloga e Mestre em Psicologia pela Universidade São Francisco. Atualmente é aluna de Doutorado em Psicologia pela mesma instituição. Membro do GT de Pesquisa em Avaliação Psicológica da Associação Nacional de Pesquisa e Pós-Graduação em Psicologia (ANPEPP). Atua em pesquisas na linha da Avaliação Psicológica e Educacional, sob os temas da motivação e autorregulação para aprendizagem, habilidades linguísticas e metalinguísticas, adaptação ao ensino superior e motivos para a evasão acadêmica.

E-mail: adrianasatico.as@gmail.com

(2) http://orcid.org/0000-0002-9856-0094

Fabián J. M. Rueda é Psicólogo e Doutor em Psicologia pela Universidade São Francisco (USF). Atualmente, é professor na Universidade São Francisco (USF) e no Programa de Pós-Graduação Stricto Sensu em Psicologia do Centro Universitário de Brasília (UniCEUB). Bolsista produtividade $1 C$ do CNPq.

E-mail: marinfabian@gmail.com

http://orcid.org/0000-0001-5173-0802 